\title{
Potential and limitations of a teleteaching environment based on H.323 audio-visual communication systems
}

\author{
Pantelis Balaouras*, Ioannis Stavrakakis, Lazaros Merakos \\ Communication Networks Laboratory (CNL), Department of Informatics, University of Athens (UoA), 15784 Athens, Greece
}

\begin{abstract}
The effective support of teleteaching services requires the development of multimedia collaboration systems that are capable of providing real-time and high quality audio-visual communication among distributed instructors and students. In the absence of such specialised systems, technologies tailored to other services are being considered for teleteaching services as well. Such a technology is the H.323 audio-visual communication technology developed to support video communication over IP. Although teleteaching and videoconferencing have similar QoS requirements, teleteaching functional requirements are a superset of those of videoconferencing. In this paper, the suitability of H.323 technology and currently available products to support teleteaching services is investigated, based on experience gained during a related deployment at the University of Athens. (c) 2000 Elsevier Science B.V. All rights reserved.
\end{abstract}

Keywords: Teleteaching environment; H.323; Multipoint conferences; IP based teleteaching; Synchronous

\section{Introduction}

Teleteaching services are expected to enable the synchronous participation in a lecture of a geographically dispersed set of students. The participants would be located in geographically dispersed facilities such as lecture theatres, classrooms, seminar rooms, laboratories, or at home. Teleteaching is expected to improve the quality of teaching especially in cases where an expert instructor is required. Another major fact that drives the deployment of teleteaching services is that the instructor's personality and instructional talent

\footnotetext{
${ }^{*}$ Corresponding author.

E-mail addresses: p.balaouras@di.uoa.gr (P. Balaouras), istavrak@di.uoa.gr (I. Stavrakakis), merakos@di.uoa.gr (L. Merakos).
}

cannot be substituted by any virtual environment or asynchronous telelearning service.

Networked multimedia applications and systems are required to implement teleteaching scenarios. Several teleteaching scenarios have been implemented in Europe by employing products based on MJPEG and ATM standards $[11,18]$.

The proliferation of IP technology has provided a widely available networking infrastructure that can easily - and in a cost effective manner - reach potential students. For this reason, it is meaningful to try to develop teleteaching services over IP, particularly now that the QoS provision capabilities of IP are close to materializing.

Two different application suites and architectures exist for multimedia collaborations over IP. The Mbone tools [12] and the H.323 audio-visual communication systems [1]. While the Mbone tools provide for a cost effective, medium quality 
audio-visual communication, mainly for the desktop, they are not mature enough to support a teleteaching environment, especially in a classroom setting. Currently available H.323 products appear to be mature for collaboration services and thus, could be used to support teleteaching services as well. Such a consideration should be investigated carefully though, the functional requirements of teleteaching can be very different from those of videoconferencing or of a simple collaboration environment.

Presently, the Communication Networks Laboratory (CNL) of the University of Athens (UoA) participates in two national projects $[16,17]$, funded by the Greek Ministry of Education and the European Commission, which aim at evaluating and exploring the potential of teleteaching scenarios based on H.323 systems. As part of these projects, the deployment of a teleteaching environment based on H.323 systems in the Athens metropolitan area is in progress.

An overview of the H.323 recommendation [1] is presented in Section 2, focusing on the multimedia stream exchange and processing capabilities of H.323 entities. The specification that is currently supported by $\mathrm{H} .323$ products is identified and discussed in Section 3. Section 4 discusses how various H.323 components may support several teleteaching scenarios and some design limitations are identified. A set of functional requirements for an effective teleteaching environment are presented in Section 5, where the limitations of present H.323 products are discussed and some solutions to overcome these limitations are provided. Finally, some enhancements to present solutions are suggested in Section 6 , in order for the supported teleteaching scenarios to better meet the functional requirements of a teleteaching environment.

\section{The ITU-T H.323 recommendations}

The International Telecommunications Union (ITU) has developed the H.323 set of standards for audio-visual and data real-time communication over IP networks [1].
The H.323 recommendations define a model that includes four types of H.323 entities, each having a particular role: the H.323 terminal, the H.323 Multipoint Control Unit (MCU), the Gateway (e.g., H.323 to H.320 Gateway), and the H.323 Gatekeeper (GK). The first three types are characterised as H.323 end-points. An H.323 endpoint generates and/or terminates streams and can call other entities and is called. An overview of ITU-T H.323, and a description of the role of each H.323 entity type is presented in [14].

In this section, we focus on the capabilities of the H.323 entities for audio, video and data stream exchange and processing; control issues at the functionality level and device control.

\subsection{Capabilities of H.323 terminals}

The ITU-T H.323 recommendations define the following capabilities of H.323 terminals.

\subsubsection{Audio issues}

All H.323 terminals must have an audio codec. An H.323 terminal may optionally send more than one audio channel at the same time and receive more than one audio channel at the same time, which may be necessary for distributed multipoint conferences. In these cases, the H.323 terminal may need to: (a) support a multicast communication mode and (b) perform an audio mixing function in order to present a composite audio signal to the user.

Each H.323 terminal should be capable of encoding and decoding speech according to Recommendation G.711, and be capable of transmitting and receiving A-law and $\mu$-law coded audio streams. A terminal may optionally be capable of encoding and decoding speech using Recommendations G.722, G.728, G.729, MPEG1 audio, and G.723. Table 1 presents the mandatory and optional capabilities of H.323 terminals.

The H.323 terminal should be capable of asymmetric operation for all audio capabilities it has declared within the same capability set. For example, it should be able to send G.711 and receive G.728 if it is capable of both. The capability set of a terminal includes its encoding and decoding capabilities. 
Table 1

Coding schemes and number of simultaneously sending and receiving streams

\begin{tabular}{|c|c|c|c|c|}
\hline & & Audio & Video & Data \\
\hline \multirow[t]{2}{*}{ Mandatory } & \#streams & 1 & 0 & 0 \\
\hline & Coding schemes & $\begin{array}{l}\text { A-law } \\
\mu \text {-law } \\
\text { G.711 }\end{array}$ & H.261 QCIF & T.120 \\
\hline \multirow[t]{6}{*}{ Optional } & \#streams & $N>1$ & $N>0$ & $N>0$ \\
\hline & Coding schemes & G.722 & - H.261 CIF & \\
\hline & & G.723 & - H.263 SQCIF, QCIF, CIF, 4CIF 16CIF & \\
\hline & & G.728 & & \\
\hline & & G.729 & & \\
\hline & & MPEG1 audio & & \\
\hline
\end{tabular}

\subsubsection{Video issues}

The video codec is optional for H.323 terminals. The H.323 terminal may optionally send over more than one video channel at the same time, and receive from more than one video channel at the same time, which may be necessary for distributed multipoint conferencing. In these cases, the H.323 terminal may need to: (a) support a multicast communication mode and (b) perform a video mixing or switching function in order to present the video signal to the user. This function may include presenting the video from more than one terminal to the user.

All H.323 terminals supporting video communications should be capable of encoding and decoding video according to H.261 QCIF [2]. Optionally, a terminal may also be capable of encoding and decoding video according to H.261 CIF or H.263 SQCIF, QCIF, CIF, 4CIF, and 16CIF [3]. If a terminal supports H.263 with CIF or higher resolution, then it should also support H.261 CIF. All terminals that support H.263 should also support H.263 QCIF.

H.323 terminals should be capable of operating in asymmetric video bit rates, frame rates and, if more than one picture resolution is supported, multiple picture resolutions. For example, this will allow a CIF capable terminal to transmit QCIF while receiving CIF pictures.

\subsubsection{Data issues}

One or more data channels are optional. The data channel may be uni-directional or bi-directional depending on the requirements of the data application.

\subsubsection{H.245 procedures}

Each H.323 terminal uses the procedures defined in H.245 [7] for

(a) exchanging its decoder capability set (video and audio bit rate, audio and picture format and algorithm options that can be accepted by the decoder), and

(b) indicating how many simultaneous audio and video streams it is capable of decoding.

The encoder of a terminal is free to transmit anything that is within the decoder's capability set.

\subsection{H.323 multipoint conferences}

The H.323 Recommendations define the multipoint controller (MC) and multipoint processor (MP) to support multipoint conferences.

\subsection{1. $M C$ and $M P$}

The MC provides control functions to support multipoint conferences between three or more H.323 endpoints, and point-to-point conferences that may become a multipoint conference. The MC may provide conference control functions such as chair control, video selection and video broadcast. The MC does not perform mixing or switching of audio, video and data streams.

The MP receives audio, video, and/or data streams from the endpoints involved in a centralised or hybrid multipoint conference. The MP processes these media streams and returns them to the terminals either in point-to-point or in multicast mode, provided that the MCU and terminals are capable of communicating in multicast mode. 
The MP may perform video switching or mixing, audio mixing, T.120 data distribution [5] and transcoding between different audio, video and data formats and bit rates in order to support conferences between H.323 terminals with different communication modes.

\subsubsection{Types of multipoint conferences}

The H.323 standard supports three types of multipoint conferences: centralized, decentralized, and hybrid (Fig. 1).

In a centralized conference, an MCU is required. The MC and MP are located at the MCU. The MC controls the conference. Each terminal transmits its video, audio, and data streams to the MCU. Then, the MP processes the streams, and distributes them back to the terminal either in unicast (point-to-point) mode or multicast mode, provided that the MCU and terminals are capable of communicating in this mode.

In a decentralized conference, each terminal multicasts its audio and video streams to all the workspaces, eliminating the need to engage a central point. The MC provides control functions and may be located at any type of endpoint. Since the terminals receive the multicast audio and video streams, an MP has to be located at the terminals to perform audio mixing and select one or more video streams to be displayed.

A hybrid conference combines elements of both a centralized and a decentralized conference. In a hybrid multipoint, centralized-audio conference, centralized multipoint is used for audio and decentralized multipoint for video. In a hybrid multipoint, centralized-video conference, centralized multipoint is used for video and decentralized multipoint for audio.

Support of centralized multipoint conferences is mandatory for H.323 terminals and MCUs; the capability to support decentralized and hybrid conferences are optional. Table 2 summarises the characteristics (capabilities, communication modes and potential locations of MC and MP(s)) for each multipoint conference type.

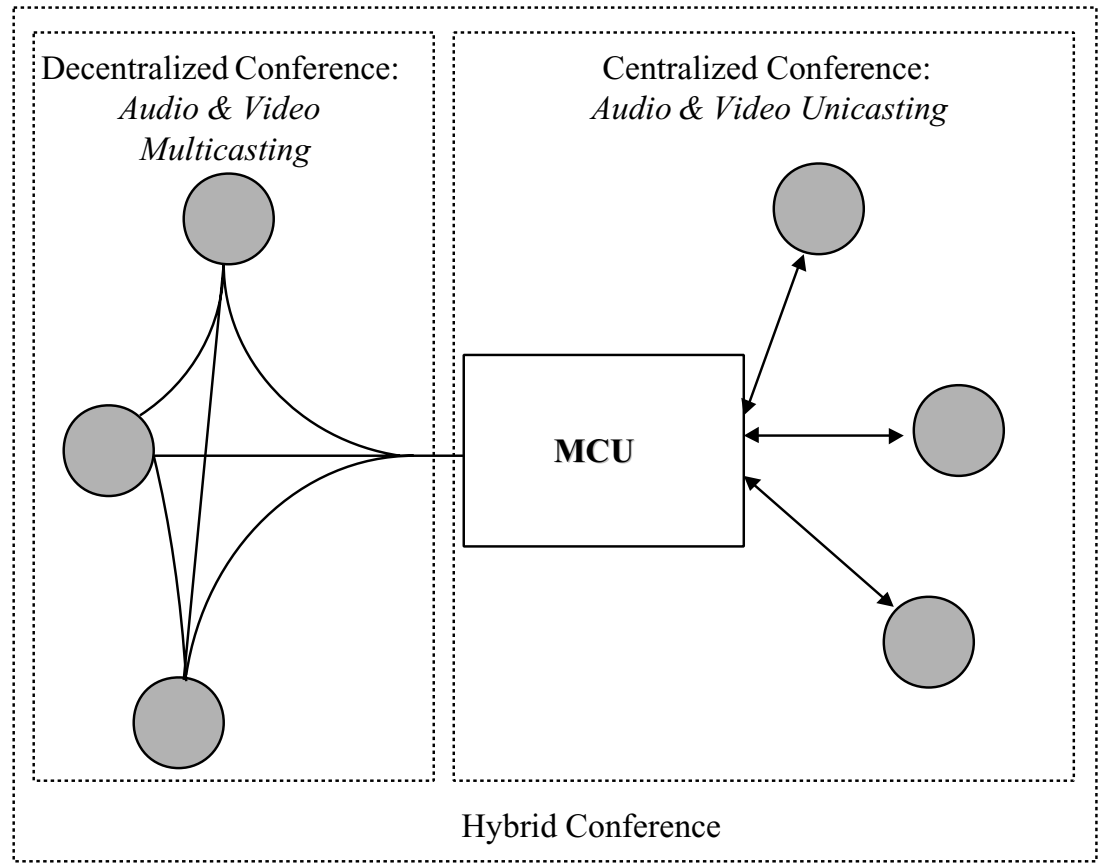

Fig. 1. Centralized, decentralized and hybrid conferences. 
Table 2

Summary of multipoint conference types characteristics

\begin{tabular}{lllll}
\hline Conference type & Centralized & Decentralized & Hybrid-centralized audio & Hybrid-centralized video \\
\hline $\begin{array}{l}\text { Capability } \\
\text { Communication modes }\end{array}$ & $\mathrm{M}^{\mathrm{b}}$ & $\mathrm{O}^{\mathrm{b}}$ & $\mathrm{O}$ & $\mathrm{O}$ \\
$\begin{array}{l}\text { Audio } \\
\text { Video }\end{array}$ & $\mathrm{P} 2 \mathrm{P}$ & Multicast & $\mathrm{P} 2 \mathrm{P}$ & Multicast \\
$\begin{array}{l}\text { T.120 data } \\
\text { Potential locations of }\end{array}$ & $\mathrm{P} 2 \mathrm{P}$ & Multicast & Multicast & P2P \\
MC & $\mathrm{PCU}$ & $\mathrm{P} 2 \mathrm{P}$ & $\mathrm{P} 2 \mathrm{P}$ \\
$\mathrm{MP}$ & $\mathrm{MCU}$, terminal, & MCU & MCU \\
& $\mathrm{MCU}$ & GK, Gateway & MP-audio: MCU & MP-audio: terminals \\
& & Terminals & MP-video: terminals & MP-video: MCU \\
& & & T.120 MP: all endpoints & T.120 MP: all endpoints \\
\hline
\end{tabular}

${ }^{a}$ Capability of Terminals and MCUs to support the conference type.

${ }^{\mathrm{b}} \mathrm{M}$ - mandatory, $\mathrm{O}$ - Optional.

\subsection{Capabilities of the H.323 MCU}

As indicated earlier, a typical MCU supports centralized multipoint conferences and consists of an MC and an MP. The MP performs video switching or mixing, audio transcoding and mixing, and T.120 data distribution.

Each terminal transmits its audio and video streams to the MCU. The MP performs synchronisation and audio mixing of the received audio streams and distributes back in point-to-point mode the mixed audio stream to each participant. The MP performs either video mixing, if supported by the MCU, or video switching, activated by voice or a chair control mechanism (Fig. 2). In the case of video mixing, the mixed stream is trans- mitted in point-to-point mode to each participant. In the video switching case, the speaker's video stream (Sa in Fig.2) is transmitted to each participant, while simultaneously the speaker's terminal may be receiving another video stream $(\mathrm{Sb}$ in Fig 2).

The MC provides conference control functions, which are discussed below.

\subsection{Control issues}

\subsubsection{Chair control}

The chair control procedure is defined in H.243 [6]. For the chair control feature to be used in a multipoint conference, both the H.323 terminals and the MCU should support this option. The

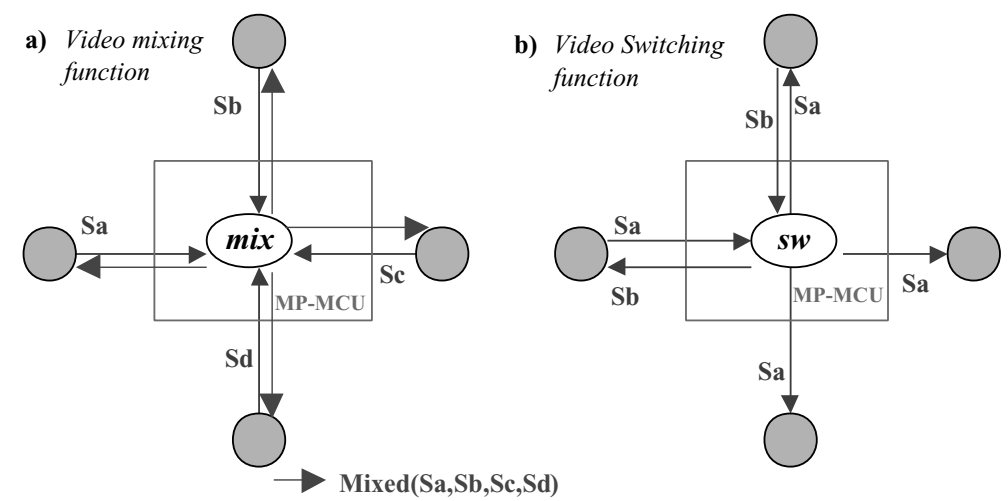

Fig. 2. Switching and mixing processing functions performed by a MP located at MCU. 
chairperson is able to manage the conference (e.g., drop participants) and select, which video stream is transmitted from the MCU to each video capable terminal. More specifically, the chair control terminal in an H.323 conference has the following visualisation-forcing capabilities:

- video broadcast, to determine which one of the available video streams will be transmitted to all terminals, except from the instructor's terminal;

- multipoint visualisation, to broadcast the chair terminal's video to all participating terminals;

- video select, to determine which video stream should be transmitted to the chair terminal.

The chair control terminal receives the floor requests of the other terminals.

\subsubsection{Floor control}

Floor control is possible only if chair control is activated. If instead of chair control, voice-activated switching or video mixing is used, then the conference is in open conversation mode, in which everyone can speak and their audio will be received by all participants.

\subsubsection{Camera control}

Camera control is dealt with in the H.281 standard [8]. A moderator, who may be either the instructor or a technician, is able to control both the local and remote cameras that are connected to an H.323 terminal that supports far-end camera control (FECC).

\section{Capabilities of present $\mathbf{H . 3 2 3}$ products}

\subsection{H.323 terminals}

Today, H.323 terminals are classified by the market as desktop and room-based systems. Both types are capable of sending and receiving one audio and one video stream, but only in pointto-point communication mode. Multicast mode is not supported yet.

The H.323 terminals may display the local or receiving video stream in small, medium or full screen window mode. "Picture in picture" (PIP) window mode is supported for a second video signal.

In Table 3, the technical profiles of currently available H.323 room-based and desktop systems are summarised [19-21].

For the transmission of the instructor's presentation data (e.g., PowerPoint presentation, PostScript slides, documents, etc.) to the remote terminals, the H.323 terminals provide application sharing services based on the ITU-T T.120 series of recommendations.

Table 3

Technical profiles of H.323 room-based and desktop terminals

\begin{tabular}{lll}
\hline Characteristics & H.323 room terminals & H.323 desktop terminals \\
\hline Audio and video inputs & $\begin{array}{l}1 \times \text { Audio, } 2 \times \text { S-video, } 1 \times \\
\text { composite }\end{array}$ & $\begin{array}{l}1 \times \text { Audio, } 1 \times \text { S-video or composite, } 1 \times \text { Composite or } \\
\text { proprietaty }\end{array}$ \\
Selection function in video inputs & Yes & \\
Audio coding schemes & $\{$ G.711,G.722,G.723,G.728 $\}$ & \\
Video coding schemes & $\{$ H.261 Sub-QCIF, H.261 QCIF, H.263 Sub-QCIF, H.263 QCIF, H.263\} \\
Type of sessions & Centralized by H.323 MCU \\
Comm. mode & point-to-point & \\
Display windows & Small, medium, full screen, PIP modes \\
Outputs & Audio RGB s-video & Audio RGB \\
Far-end camera control & Yes & Some \\
Floor control & Only via chair control & No \\
Chair control & Yes & No \\
Display devices & TV & PC monitor PC speakers \\
Data support & Yes, by T.120 application sharing services \\
Underlying network technology & IP, ISDN, MVIP & IP and some ISDN \\
Platform & PC based Windows & \\
\hline
\end{tabular}




\subsection{H.323 MCUs}

Today's H.323 MCU products support only the centralized multipoint conference type, which is defined as mandatory by the H.323 recommendations. The H.323 MCU products support the distribution of audio and video streams only in point-to-point mode and not in multicast mode, resulting in a waste of network bandwidth. H.323 MCUs are presently supporting voice activated video switching, audio transcoding and mixing, but not video mixing yet. The chair control function is also supported.

\subsection{Quality of multipoint conferences}

The quality of a multipoint conference depends on the audio and video coding standards supported by the H.323 terminals, the stream bit rate, and the capabilities of the H.323 MCU to support switching of multiple high bit rate video streams.

\subsubsection{Control issues}

Only H.323 room-based terminals support chair control. In the case of centralized multipoint conference, chair control may be enabled, provided that the H.323 MCU supports it.

Camera control is provided by all H.323 roombased terminals and some of the desktop terminals.

\section{Teleteaching environment}

A teleteaching environment is expected to enable the synchronous participation in a lecture of a geographically dispersed set of students. The participants could be located in geographically dispersed facilities such as lecture theatres, classrooms, seminar rooms, and laboratories or at home. These facilities are referred to as teleteaching workspaces.

A teleteaching session $i$ (TSi) will involve a number of workspaces and necessitate certain interactions among them. Let Wspaces (TSi) denote the set of these workspaces. The interactions between the workspaces constitute the scenario of the teleteaching activity, referred to as the teleteaching scenario.
Table 4

Notation; the argument indicates the associated workspace

\begin{tabular}{ll}
\hline Notation & Description \\
\hline$W i$ & Instructor's workspace \\
$\mathrm{MS}$ & Media server \\
$\mathrm{Iv}(W)$ & Instructor video signal \\
$\mathrm{Ia}(W)$ & Instructor audio signal \\
$\mathrm{Sv}(W)$ & Student(s) video signal \\
$\mathrm{Sa}(W)$ & Student(s) audio signal \\
$\mathrm{Dp}(W)$ & Data presentations \\
$\mathrm{Dv}(W)$ & Pre-recorded video stream in H.263, \\
& MPEG1 or MPEG2 format \\
$\mathrm{Da}(W)$ & Pre-recorded audio stream, e.g., mp3 \\
& streams \\
$\mathrm{D}(W)$ & Represents one of Dp, Dv and Da \\
$\mathrm{V}(W)$ & Visual presenter video signal \\
$\mathrm{Mv}(W)$ & VCR video signal \\
$\mathrm{Ma}(W)$ & A tape or CD audio signal \\
\hline
\end{tabular}

Our aim is to present the set of interactions (i.e., the potential teleteaching scenarios), which can be supported by present H.323 products as well as identify the limitations of these products. For this purpose, a description of the available signals and teaching media in a teleteaching workspace is needed.

In the interest of saving space the standard equipment (capture and display devices, videoconference systems) of a typical teleteaching workspace are not described here. Such a description may be found in [10]. A notation concerning the teaching media sources, in analogue (outputs of capture devices) and digital forms is provided in Table 4. This notation is used in this paper.

Fig. 3 illustrates the devices associated with the analogue and digital teaching media presented in Table 4.

\subsection{Required H.323 entities}

It is assumed that only currently available H.323 audio-visual communication systems are used to implement a teleteaching activity.

In addition to the H.323 terminals, an H.323 MCU and an H.323 to H.320 Gateway are required to deploy a teleteaching scenario based on H.323 and H.320 [4] audio visual communication systems (Fig. 4). An H.323 Gatekeeper may optionally be used as well. 


\section{Teaching media Input Devices}

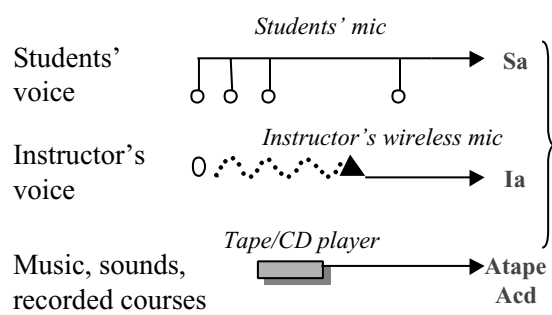

\section{Teaching media Input Devices}

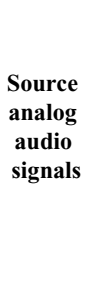

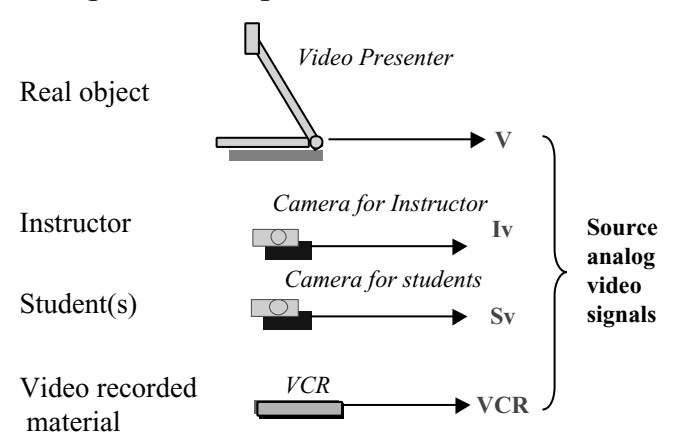

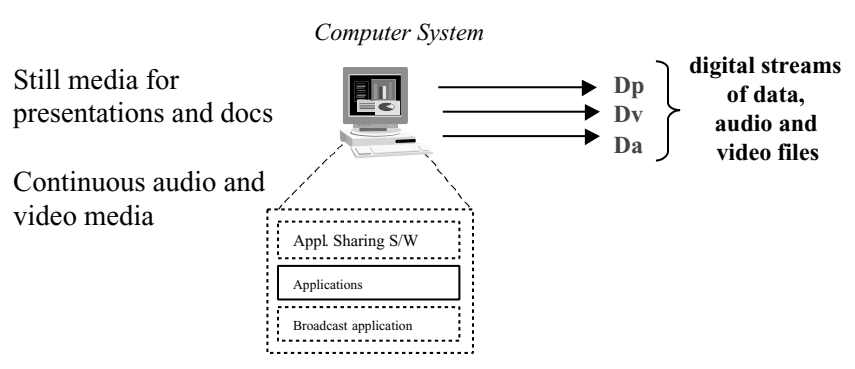

Fig. 3. Teaching media and associated devices.

A workspace $W$ may be equipped with one or more H.323 terminals depending on the teleteaching scenario.

For instance, multiple H.323 terminals are necessary if multiple streams (audio or video) are expected to be transmitted from workspace $W$. It is reminded that an H.323 terminal supports the transmission of only one audio and one video stream. In a typical teleteaching scenario where both the instructor's and the student's figures and voices need to be transmitted from a workspace, two H.323 terminals are usually installed in the instructor's workspace: one for transmitting the instructor's audio and video, and one for transmitting the students' audio and video.

In the case in which only one H.323 room-based terminal is installed in a workspace, both the instructor's and student's cameras are connected to a single H.323 terminal. A visual presenter may be connected as well, since room-based terminals are equipped with three video inputs. Only one video input is selected as video source each time. If additional video sources exist, such as a VCR, then a video matrix has to be installed in the workspace as well.

\subsection{Potential interactions among workspaces}

In the following paragraphs, the potential interactions between the workspaces, which can be supported by currently available H.323 products, are discussed. Fig. 5 provides an illustration of stream exchange.

In workspace $W i$, the instructor's camera and a wireless microphone are connected to an H.323 room terminal; let $\operatorname{Sys} 1(\mathrm{Wi})$ denote this terminal. The appropriate viewer for the data presentation(s) should be installed in Sys1(Wi). The instructor's video $\operatorname{Iv}(W i)$, audio $\operatorname{Ia}(W i)$ and data presentation $\mathrm{Dp}(W i)$ streams are transmitted to the H.323 MCU from Sys1(Wi). The V(Wi) visual presenter signal could be transmitted, instead of $\operatorname{Iv}(W i)$. The student's camera and microphone are connected to the second H.323 room-based terminal; let Sys2( $W i)$ denote this terminal. Student's video $\operatorname{Sv}(W i)$, and audio $\mathrm{Sa}(W i)$ are transmitted to the H.323 MCU as well. 


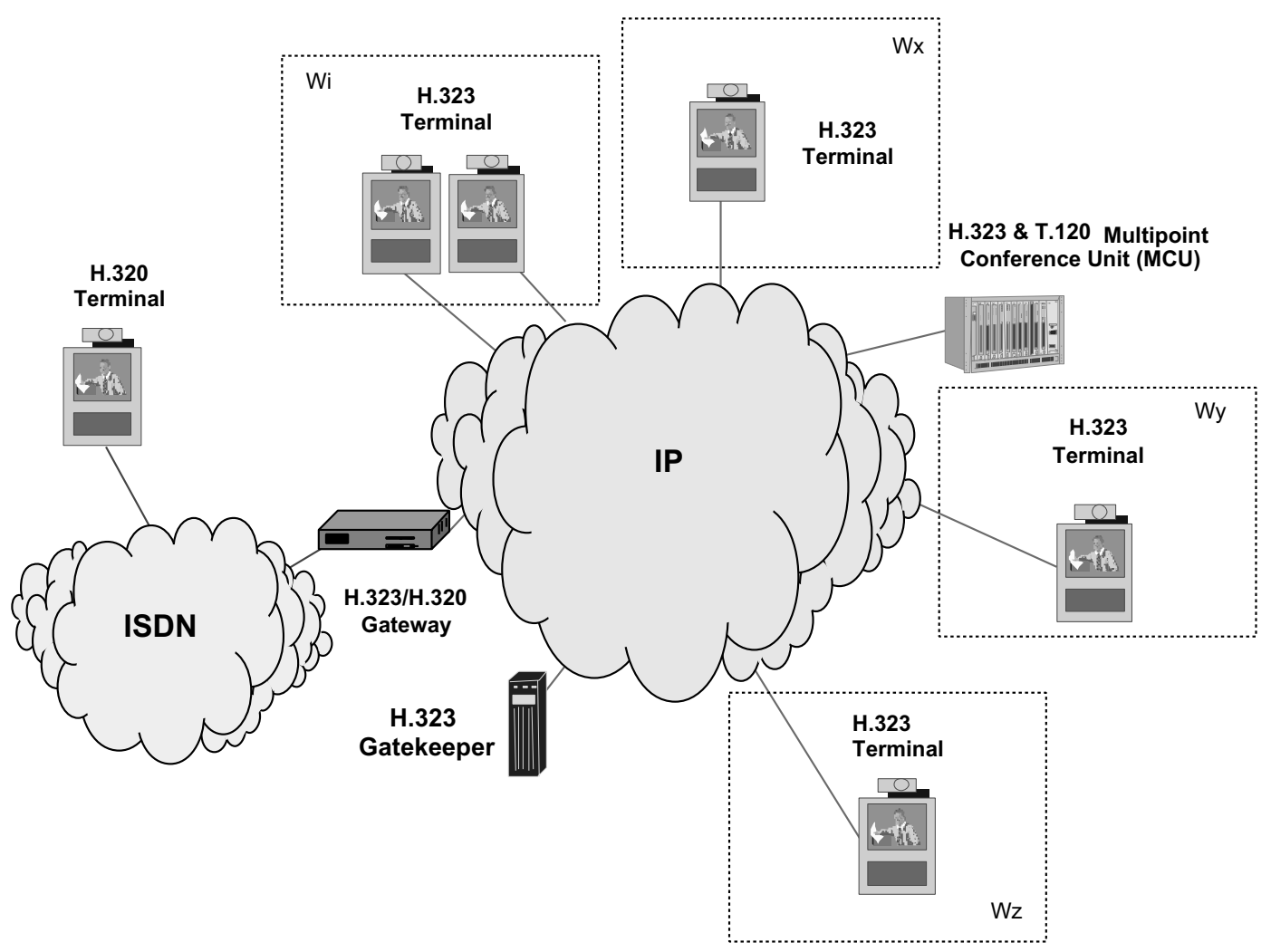

Fig. 4. H.323 and H.320 components of a teleteaching environment.

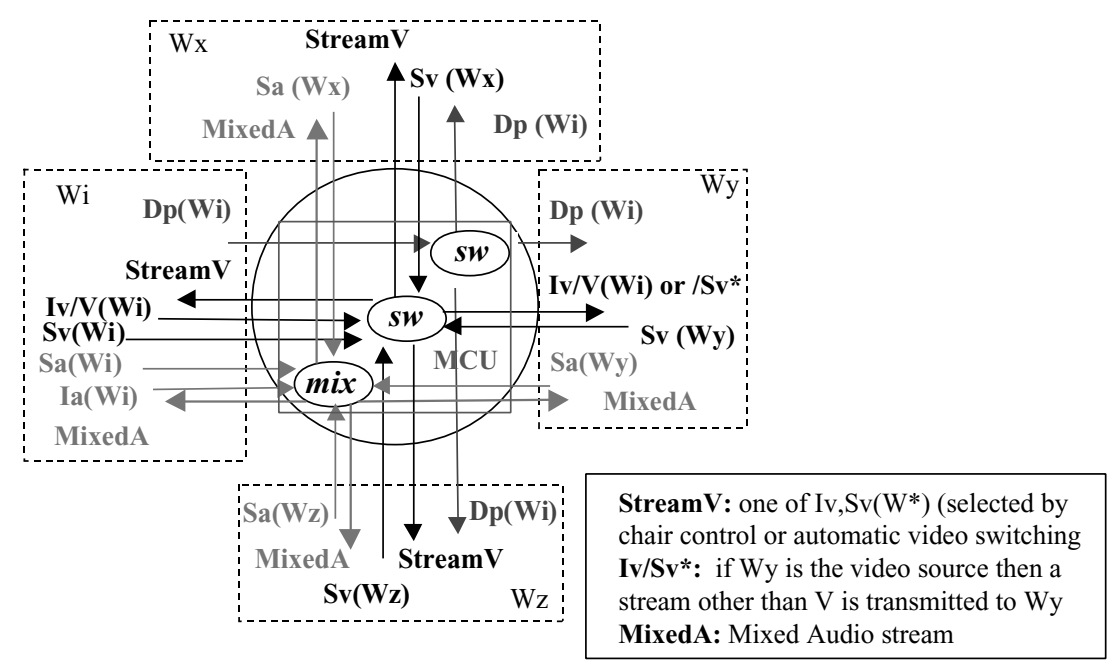

Fig. 5. Stream exchange among the workspaces in the H.323 teleteaching scenario. 
In each of the remote workspaces $W x, W y, W z$, a single H.323 terminal is assumed to be installed; let $\operatorname{Sys} 1(W)$ denote this terminal. The student's camera and microphone are connected to $\operatorname{Sys} 1(W)$. The student's video $\operatorname{Sv}(W)$, and audio $\mathrm{Sa}(W)$ are transmitted to the H.323 MCU as well.

In summary, the H.323 MCU receives the set of video streams Vset $=\{\operatorname{Iv}(W i), \operatorname{Sv}(W i), \operatorname{Sv}(W x)$, $\operatorname{Sv}(W y), \operatorname{Sv}(W z)\}$, the set of audio streams Aset $=$ $\{\mathrm{Ia}(W i), \mathrm{Sa}(W i), \mathrm{Sa}(W x), \mathrm{Sa}(W y), \mathrm{Sa}(W z)\}$, and the data stream Dp.

Current H.323 MCU products are capable of performing the following functions:

- Audio mixing of audio streams and distributing the produced stream, denoted as MixedA (Fig. 5), back to each workspace in point-topoint mode.

- Video switching one video stream from the set Vset is selected and transmitted back to the H.323 terminals in point-to-point mode. Let StreamV denote this stream. There are two ways for selecting the stream. An automatic way, in which a voice-activated mechanism selects the speaker's video for transmission. Alternatively, the chairperson selects which video stream should be transmitted to the remote terminals, provided that H.323 room systems and MCU support chair control.

- Distribution of the data stream Dp to all the remote terminals in point-to-point mode.

Thus, each participating H.323 terminal receives three streams: the audio stream MixedA, the video stream StreamV and the data stream Dp. The stream MixedA is decoded and channelled to the speakers, while StreamV and Dp are displayed on one or two monitors. The processing functions taking place in the MCU, and the streams distributed by the MCU to the terminals of the workspaces are illustrated in Fig. 5, for the case of automatic video switching.

\section{Functional requirements}

In this section, a set of teleteaching functional requirements is presented and the degree to which, currently available H.323 products fulfil these requirements is examined. For the requirements that are partly or unfulfilled, some solutions are proposed.

\subsection{Lecture delivery phase requirements}

The main phase of a teleteaching activity is the lecture delivery phase, in which a remote instructor lectures. The major requirement $(R l)$ is that the remote students should have high quality audiovisual communication with the instructor and his/ her presentation data.

This communication requirement is fulfilled since present H.323 MCU products support the chair control mechanism, which controls and enables the constant selection of $\operatorname{Iv}(W i), \operatorname{Ia}(W i)$, and $\mathrm{Dp}$ streams for switching to all remote workspaces. The participating H.323 terminals should support chair control. Automatic video switching mode can be used, but in this mode interference from another participant is possible.

To meet the high-quality requirement, G.711 audio coding (64 kbps) should be used, and the video codec should support $30 \mathrm{fps}$ CIF at a minimum of $768 \mathrm{kbps}$.

As far as the display of $\mathrm{Dp}(W i)$ and $\operatorname{Iv}(W i)$ is concerned, it is often required that each of them be displayed on a separate display device $(R 2)$. In the case that a workspace is equipped with only a single display device, Dp should be displayed in full screen mode and $\operatorname{Iv}(W i)$ in a PIP window, since, from an educational perspective, it is considered that the data presentation has higher priority than the instructor's video. The display of video and data presentations in dual monitors is currently supported by many H.323 terminals.

A third requirement $(R 3)$ often met is that instructors should be free to move around as in a conventional classroom. To enable this, the instructor's camera should be a Pan-Tilt-Zoom camera in order to follow him/her as he/she moves. A wireless microphone should also be used by the instructor to facilitate his/her movement. The instructor's camera could support autotracking as well. In this case, no cameraman or technician is required for the camera control. 


\subsection{Instructor's interaction with students}

Instructors interact with students, when students ask questions or participates in a conversation with the instructor. In this state of a teleteaching interaction, all remote participants should have audio and visual (less priority in comparison to audio) contact with the person participating in the dialogue with the instructor $(R 4)$. This requirement can be fulfilled in two ways:

1. By operating the MCU in the automatic video switching mode. Due to the audio mixing, the participants listen to the dialogue immediately. The visual contact is realised after a person continuously speaks for two seconds. This time is required in order for the MCU to determine who the speaker is, and switch the speaker's video stream to the remote participants.

2. By operating the MCU in the chair control mode. In this mode the chairperson grants the floor to the person speaking and their workspace terminal. The audio and video stream is then switched immediately to all participants. A major problem arises when a student/participant wishes to ask for the floor (R5). Floor control and request is enabled only in the chair control mode. Only the operator of the H.323 room terminal is able to request the floor. Thus, the operator has to request the floor on behalf of a student and this may discourage the student's active participation in the lecture.

A complementary floor control system could also be used. This system should co-operate with both the H.323 terminal and the camera system. The floor control system should permit the student to submit and cancel floor requests to the terminal. The chairperson should be able to view the request and decide to whom to grant the floor. When the floor is granted to a student, the camera system should tilt and zoom to that student.

If chair control is not supported by the terminals, then social rules, e.g., hand raising, has to be employed.

\subsection{Open conversation}

In certain circumstances, support of open conversation between all the participants is required
$(R 6)$. The audio mixing function permits the open audio conversation of the participants. The MCU selects for switching the video stream of the participant who speaks for more than two seconds.

This requirement is fulfilled by using the microphone system in "momentarily on" mode. In this mode, each speaker should touch the microphone's button while speaking. When the button is released the microphone is muted and the audio stream does not contain audio samples. Thus the MCU recognises the current speaker and selects his or her video.

\subsection{Interference of an external person}

The teleteaching environment should allow for the participation and intervention of an external person in the teaching process $(R 7)$. This requirement can also be fulfilled. The person should use either an H.323 or H.320 terminal and an H.323/ H.320 Gateway, and participate just as another workspace.

\subsection{Projection of the remote workspaces' signals}

A requirement $(R 8)$ often set by pedagogical experts is the following one: the students should be able to see the reactions and gestures of the remote classroom students in order to avoid sensory deprivation [9]. This requirement could be fulfilled by displaying the remote workspaces' video in one of the permanent, periodic or instant display modes. The permanent display requires the installation of a display device in each workspace, dedicated to permanently displaying the remote workspaces. The periodic and instant display of remote workspaces could be done in a single monitor. In all modes, either one workspace each time, or a panorama of all or some of the workspaces could be displayed.

Today, no simultaneous view of the instructor's video and anyone's remote workspace is feasible either on the main or dual monitor. This is because neither the video mixing function is supported by current H.323 MCU products nor is the capability to receive multiple video streams supported by present H.323 terminals. For these reasons, only the periodic or instant display of remote students 
can be implemented today with current H.323 technology. The chair control capability is used for this purpose. The chairperson, periodically or instantly, selects a specific workspace's video stream. For the implementation of a permanent display, other technologies, such as the Mbone tool, vic [12], have to be used.

In the future, H.323 products could support this teleteaching requirement by enhancing the MCUs and terminals. There are two alternatives, depending on whether a single or multiple workspaces' video(s) should be displayed simultaneously:

A. Multiple workspaces

1. The H.323 MCUs have to concurrently support:

- video switching of the current speaker's video (e.g., the instructor's);

- video mixing of the received streams and switching of the produced stream to the H.323 terminals.

2. The H.323 terminals have to be capable of:

- receiving two video streams (the speaker's and the workspaces');

- displaying each video stream on a separate monitor.

B. Single workspace

1. The H.323 MCUs have to concurrently support selection and switching of two video streams: the speaker's and a workspace's.

2. The H.323 terminals have to be capable of:

- receiving the two video streams;

- displaying each video stream on separate monitor.

5.6. Projection of the remote classroom on the instructor's small size monitor $(s)$

It is sometimes desirable for the instructor to be able to permanently attend the workspaces of students located at remote sites by displaying each workspace's video signal in a small size monitor $(R 9)$. This requirement is similar to the previous one.

\subsection{Lecture broadcasting}

It should be possible to broadcast a lecture to a large number of students, who should be able to receive the lecture but not interact (passive students) (R10). Currently, this requirement is not fulfilled. A limited number of users - each equipped with H.323 terminals - are permitted to be connected to an MCU. Lecture broadcasting service could be provided by MCUs capable of multicasting the audio, video, and data streams. The passive users should be able to use an H.323 terminal capable of joining the multicast session. If the MCU transcodes the video by employing a low quality coding scheme (e.g., Sub-QCIF), then the H.323 terminal could be only a software entity.

An alternative is to implement a broadcasting entity, which participates in an H.323 multipoint centralized conference as an H.323 terminal, and multicasts the receiving streams to the passive participants. The participants may use either the Mbone applications [13] or a commercial product. An H.323 product that follows this approach exists, but it only communicates with a specific commercial product.

\subsection{Session recording}

It should be possible to record a teleteaching session and make it available for latter asynchronous access by the students or the instructor $(R 11)$. This is not supported by the H.323 recommendation. A complementary system should be used, possibly in conjunction with the broadcasting system.

\subsection{Session management}

A teleteaching environment should provide a way for session announcement, scheduling and management (R12). The active participants should be invited by a session control entity, while passive participants should be able to join the session. The requirement for an authentication mechanism is optional. Statistics should be kept in a database for billing and accounting.

Currently, the administrator of an H.323 MCU is able to schedule a conference by inserting the IP addresses of the potential participants in the MCU. An H.323 terminal whose IP address is not included in the IP address list cannot join the conference. No announcement mechanism is pro- 
vided by the H.323 recommendations for announcing the session. Usually, e-mail communication is used for announcing the schedule to potential participants.

In current multipoint centralized conferences, the participants have to call the MCU. No invitation procedure from the MCU to the participants is supported. Statistics are not kept for billing, accounting or monitoring usage.

\section{Conclusions}

Current H.323 products are suitable for deploying point-to-point and multipoint videoconference services and collaboration over IP networks in a business environment; however, as far as teleteaching services are concerned, present H.323 products can only partly fulfil the requirements.

H.323 products need to be enhanced in order to support high-quality teleteaching services. More precisely H.323 MCUs should be enhanced to:

- support the multicast communication mode for stream distribution;

- control the terminals so that they do not transmit their video stream when they are not selected by the H.323 MCU for switching (currently not always available);

- support the video mixing function;

- support the video transcoding function;

- allow for switching of multiple video streams (at least two video streams);

- enable concurrent video mixing and switching.

The H.323 terminals should be enhanced to:

- support the multicast communication mode;

- be capable of receiving and decoding at least two video streams, and displaying them in dual monitors;

- support chair control (desktop terminals).

All the above capabilities are defined by the H.323 recommendation. An important enhancement, which is not defined by the H.323 recommendation, is the definition of the procedures that will permit the co-operation of room-based systems with microphones and camera systems. A microphone system should provide capabilities for submitting and cancelling floor request. The camera system should be able to tilt and zoom to the person who is granted the floor.

Assuming that $\mathrm{H} .323$ products will support these enhancements, the question that remains is how cost-effective and flexible is the deployment of teleteaching services based on H.323 audio visual systems compared to alternative technologies and architectures such as the Mbone tools and scalable and composable architectures for distributed multimedia collaboration such as the MASH architecture [15]?

\section{Acknowledgements}

This work and its dissemination efforts have been supported in part by the IST Program of the European Union under contact IST-1999-10160 (VideoGateway).

\section{References}

[1] ITU-T Rec. H.323, Visual telephone systems and terminal equipment for local area networks which provide a nonguaranteed quality of service, 1996.

[2] ITU-T Rec. H. 261, Video CODEC for audiovisual services at p x $64 \mathrm{kbit} / \mathrm{s}, 1993$.

[3] ITU-T Rec. H 263, Video CODEC for narrow telecommunications channels at $<64 \mathrm{kbit} / \mathrm{s}, 1995$.

[4] ITU-T Rec. H 320, Narrowband ISDN visual telephone systems and terminal equipment, 1995.

[5] ITU-T Rec. T.120 Data protocols for multimedia conferencing, 1996.

[6] ITU-T Rec. H.243, Procedures for establishing communication between three or more audiovisual terminals using digital channels up to $1920 \mathrm{kbit} / \mathrm{s}, 1996$.

[7] ITU-T Rec. H.245, Control protocol for multimedia communication, 1997.

[8] ITU-T Rec. H.281, A far end camera control protocol for videoconferences using H.224, 1995.

[9] J. Bourdeau, M. Quallet, R. Gauthier, Interactivity in videoconference based telepresentations, ED-MEDIA \& ED-TELECOM 98.

[10] R. Grebner, Use of instructional material in universal teleteaching environments, Computer Networks and ISDN Systems 29 (1997) 1787-1797.

[11] A. Klein, Teleteaching scenarios for high bandwidth networks, Computer Networks and ISDN Systems 30 (1998) 1707-1716.

[12] S. McCanne, V. Jacobson, vic: A flexible framework for packet video, ACM Multimedia (November 1995). 
[13] S. McCanne, Scalable multimedia communication using IP multicast and lightweight sessions, IEEE Internet Computing (March-April 1999).

[14] G.A. Thom, H.323: The multimedia communications standard for local area networks, IEEE Communication Magazine (December 1996) 52-56.

[15] http://mash.cs.berkeley.edu/mash/index.html.

[16] http://www.gunet.gr/pilots/.

[17] http://www.cnl.di.uoa.gr/Projects/tuatm.html.

[18] T. Walter, H. Haenni, Telepoly - a teleteaching scenarion supported by high-speed networks, http://www.tik.ee.ethz. $\mathrm{ch} / \sim$ walter/Telepoly/Telepoly.html.

[19] http://www.vcon.com.

[20] http:// www.picturetel.com.

[21] http:// www.intel.com/proshare.

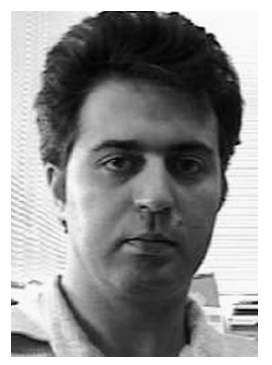

Pantelis Balaouras received his Diplo$\mathrm{ma}$ in Computer Engineering and Informatics from the University of Patras, Greece, in 1992. From 1992 to 1995, he was a Research Scientist at the Computer Technology Institute, Patras, Greece, and since 1996, he has been working as a Research Engineer at the Communication Networks Laboratory (CNL) of the University of Athens. He is a Ph.D. candidate in the Department of Informatics, University of Athens, and his research work focuses on the design, development and deployment of advanced multimedia services. He has been involved in a number of European and national networking projects. He is also a technical consultant for the Greek Universities Network (GUnet) and a member of the technical committee of the Greek National Research Network (GRnet).

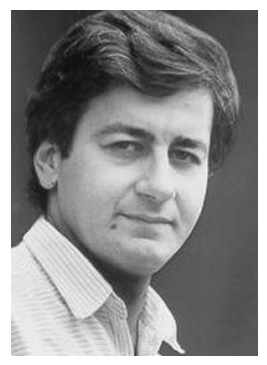

Ioannis Stavrakakis received his Diploma in Electrical Engineering, Aristotelian from the University of Thessaloniki, Greece, in 1983 and his Ph.D. in EE from the University of Virginia, USA, in 1988. He was assistant professor in CSEE, at the University of Vermont, USA, between 1988 and 1994; associate professor of ECE at Northeastern University, Boston, USA, from 1994 to 1999 and is currently, associate professor of Informatics at the University of Athens, Greece. His teaching and research interests are focused on resource allocation protocols and traffic management for communication networks. His past research has been published in over 90 scientific journals and conference proceedings.

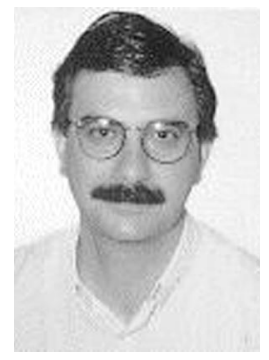

Lazaros Merakos received his Diploma in electrical and mechanical engineering from the National Technical University of Athens, Greece, in 1978, and M.S. and Ph.D. degrees in electrical engineering from the State University of New York, Buffalo, in 1981 and 1984, respectively. From 1983 to 1986, he was the faculty of Electrical Engineering and Computer Science at the University of Connecticut, Storrs. From 1986 to 1994 he was the faculty of the Electrical and Computer Engineering Department at Northeastern

University, Boston, MA. In 1994, he joined as the faculty of the University of Athens, Greece, where he is presently a Professor in the Department of Informatics, and Director of the Communication Networks Laboratory (CNL) and the Networks Management Center. His research interests include design and performance analysis of broadband networks, mobile networks, and advanced video communication technologies and services. $\mathrm{He}$ is the author of over 110 papers in the above areas. Since 1996, he has been responsible for the deployment of the Greek Universities network (GUnet), the national academic network offering high-speed connections and advanced networking services to the 18 Universities and 14 Technological Education Institutes (TEI) of Greece. 